\title{
Recuerdo histórico y comentario sobre "la epidemia tifoidea de la isla de Huar. Provincia de Llanquihue"
}

\author{
Enrique Laval $R$.
}

\section{Historic memory and commentary about "typhoid fever epidemic at Huar Island". Llanquihue province}

An article by Dr. Carlos Martin in the first published number of the Revista Médica de Chile (1872-73) journal is remembered and commented. It describes an outbreak in Huar Island in Seno de Reloncavi, an estuary located in the Los Lagos Region in Chile, named "typhoid epidemic". The clinical characteristics are presented and two types of diseases are deducted: acute respiratory infections and typhoid fever.

Key words: Epidemic, acute respiratory infections, typhoid fever.

Palabras clave: Epidemia; infecciones respiratorias agudas; fiebre tifoidea.
Pontificia Universidad Católica de Chile.

Facultad de Medicina. Programa de Estudios Médicos Humanísticos.

Recibido: 28 de agosto de 2010 Aceptado: 29 de septiembre de 2010

Correspondencia a:

Enrique Laval R.

revinf@sochinf.cl
$\mathrm{E}$ n el primer volumen de la Revista Médica de Chile, correspondiente a los años 1872-1873, se publicó la comunicación del doctor Carlos Eduardo Martin, sobre "La epidemia tifoidea de la isla de Huar, provincia de Llanquihue".

\section{Datos biográficos del doctor Carlos Eduardo Martin}

El doctor Martin era alemán, nacido en Jena el 16 de septiembre de 1838. Estudió Ciencias Naturales y Medicina en aquella ciudad y Berlín, titulándose de médico en 1861. Al año siguiente se fue a Brasil, como miembro agregado a la Embajada de Alemania. Revalidó su título con una tesis sobre la infección puerperal. El año 1864, debido a la guerra entre Alemania y Dinamarca, regresó a su patria, permaneciendo después en Berlín como asistente de su padre, el doctor Eduardo Martin, profesor de Ginecología y Obstetricia. Publicó en esa época estudios sobre la pelvimetría externa de la mujer, que más tarde le sirvieron para titularse en Chile; radicóse primero en Puerto Montt (1871) y después en Ancud, retornando definitivamente a Puerto Montt, siendo designado médico de la colonia alemana residente.

En 1876, volvió a Alemania y estableciose en Jena, escribiendo una interesante reseña sobre las epidemias de peste bubónica durante la Edad Media.

Regresó a Chile en 1884, nombrándosele médico de vacuna y del Hospital de Puerto Montt.

Sus conocimientos y estudios geográficos le permitie-

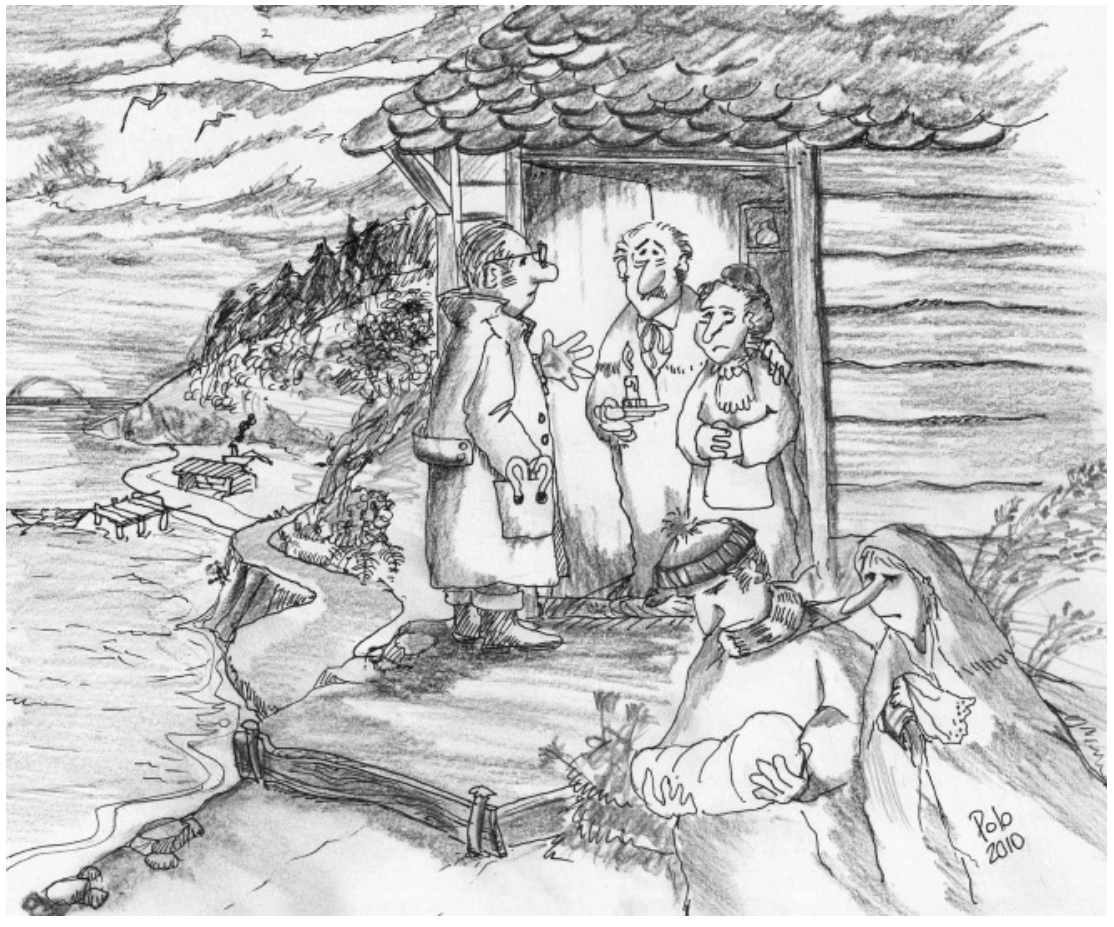

ron asesorar al árbitro ingles Lord Haldrich en problemas limítrofes con Argentina.

En 1887 era profesor de Historia Natural en el Liceo de Puerto Montt y de Higiene en la Escuela Normal, cargos que desempeñó hasta principio de 1907, falleciendo en febrero de aquel año ${ }^{2}$. 


\section{Geografía física y política de la isla de Huar}

La isla Huar (conocida también como Guar), pertenece al Departamento de Carelmapu, $X^{\mathrm{a}}$ Región de Los Lagos, a 15 millas al sur de Puerto Montt. Se encuentra bajo los $41^{\circ} 43^{\prime}$ de latitud y $72^{\circ} 50^{\prime}$ de longitud, al este de Calbuco, separada del norte de tierra firme por un pasaje hondo de dos kilómetros de ancho

Mide de este a oeste unos 9 kilómetros y unos 3 de norte a sur. Su superficie de 35 kilómetros cuadrados, está compuesta de lomajes de baja altura con suave descenso hacia el mar, a excepción de la parte que mira al sur donde es algo escarpada. Su tierra fértil permitía cultivar trigo, avena, habas, arvejas y papas; por lo general, cubierta de bosque espeso en el que se cortaba laurel, lama y tique. También se criaba ganado vacuno, lanar y porcino. Tenía agua corriente que se utilizaba como fuerza motriz en varias partes (desde 2005 la luz eléctrica era ya una realidad en la isla).

Se encuentra en la parte central del seno de Reloncaví, entre las islas Maillen y Puluqui. Deriva su nombre del indio que primero vivió allí, llamado Huercán, comenzando a poblarse en 1710, en que su dueño don Juan de Uribe la regaló a unas treinta familias de indios chonos. El Presidente don Juan Ustáriz la denominó isla de San Felipe de Guar, lo que fue aprobado por Real Cédula de 31 de julio de 1713 .

La isla se encuentra cortada por varias internaciones o esteros (los más importantes son los de Chauque, Chemcoihue y Quetrocuaquin) donde abundan pejerreyes y róbalos, así como cholgas, piures, quilmahues, ostras y navajuelas.

Si bien a fines del siglo XIX se consideraba poblada por 2.300 habitantes, a principio de 2008 se hablaba de sólo $1.400^{1,3-5}$.

\section{La epidemia}

Al presentar "estas líneas a la Sociedad Médica" el doctor Martin hace notar "que la falta de recursos cientificos en estas regiones no le han permitido hacer investigaciones exactas sobre la naturaleza de la enfermedad". Llama la atención que "es un hecho triste que los huarunos suelen gastar la ganancia del verano antes de su vuelta a la isla, así que en ésta viven en un estado miserable". Por lo demás "la chicha de manzanas agrias es casi su única bebida a no serlo el aguardiente en días de fiesta".

La iniciación del invierno del año 1872 "no comenzó con lluvias abundantes, como suele principiar, sino con unos vientos fríos del sur, muy helados, los cuales generalmente no suelen soplar, sino de julio para adelante".
La temperatura había bajado hasta 1,1 centígrado, "mientras que en ninguno de los diez años anteriores había llegado a menos de 3 centígrados".

El frío continuó seco, y con vientos australes muy frecuentes, desde marzo a mayo y ya en junio la temperatura llegó al término medio general habitual.

Continúa el doctor Martin diciendo "que la isla está mucho más abierta a los vientos australes, alternando en Puerto Montt los vientos del sur con los del norte. En cambio en la isla alternaron los del sur con los del este".

Según el autor "corresponde explicar el que en la misma punta sud-este, donde está la más expuesta a los vientos fríos, la enfermedad principiara a devastar la isla, alcanzando también allí su intensidad más mortal. Dificil sería decidir, si aqui ha tenido su origen o si ha sido importada de un puertecito cercano nombrado Lenca".

Este "puertecito", como lo llama el doctor Martin, corresponde a una caleta del departamento de Llanquihue, próxima al sur este de Puerto Montt y al lado sur de la desembocadura del riachuelo de Coiherin. Es una serie de casitas bajas de madera, agrupadas alrededor de una capilla y de una escuela pública, habitadas por "huarunos" en situación climática parecida a la isla: también está expuesta a los vientos fríos de la cordillera alta, al pie de la cual se extiende. Como hay bastante tráfico, entre la isla y Lenca, "puede también suponerse que haya recibido la enfermedad de la isla"1,3,4.

Después de extinguirse la epidemia en la isla (septiembre de 1872), afirma perentoriamente Martin, "aparecieron unos casos muy parecidos en algunas partes del continente, sin que falleciera ninguno". En la lista de muertos de la isla de Huar, "todos los casos acontecieron dentro de los meses de marzo a agosto y entre ellos cinco creaturas de menos de un año, todos débiles y también dos personas que sin duda murieron de tubérculo de los pulmones".

Dividiendo los muertos según los lugares, resulta que de los 28 causados por la epidemia, 19 fueron del distrito oriental de la isla, de nombre Onetrulanquén (761 habitantes), 7 del distrito medio, el de Alfaro (486 habitantes) y 2 del distrito occidental, Chucagua (410 habitantes), lo que constituye una nueva corroboración de los argumentos arriba mencionados: la mayor mortalidad se encuentra al este en la parte más fría, menos abrigada, más pobre y con la población más concentrada. También el distrito más devastado por la epidemia, se considera que es el que cultiva más el comercio con Puerto Montt y "por eso tiene las costumbres menos puras".

La epidemia atacó más bien a las personas enfermizas y débiles que a las robustas. Así la mitad de los enfermos había tenido mala salud anteriormente.

El primer síntoma fue la anorexia, a veces acompañada de vómitos. Al día siguiente escalofríos repetidos de mayor o menor fuerza seguidos de temperatura alta 
y continua, taquicardia, un dolor agudo, intenso, exasperante en el tórax y menos frecuente en el abdomen, sintomatología que llamó tanto la atención de los isleños que le dieron el nombre de "puntada" a la epidemia. En numerosos casos existió tos con expectoración, no muy abundante, pero sí sanguinolenta. Según el doctor Martin "en el examen toráxico se comprobó escrepitaciones húmedas, roncus y sibilancias, con focos de pulmonía lobular. La palpación abdominal demostró casi siempre hepato-esplenomegalia".

Agrega Martin que "el abdomen entero, generalmente se encontraba adolorido, un poco hinchado y blando; mostraba gorgoteo y dolor ileocecal, que caracteriza el iliotifus. La evidencia que era la epidemia de tifus me dieron estas manchitas de roseola tifoidea que comprobé en 7 enfermos examinados en el periodo avanzado de la enfermedad. La lengua al principio un poco blanca, pronto se limpiaba y mostraba ora una, ora dos rayas amarillas o morenas; sin embargo, no tenía en ningún caso el color fuliginoso que se encuentra en caso del tifo abdominal intenso. El pulso oscilaba entre 95 y 110 golpes por minuto y el alza de temperatura varió entre 38.6 y 40, 7 grados".

Sin embargo, comenta el doctor Martin, "había síntomas que establecían una diferencia notable entre nuestra epidemia y la de iliotifus. La diarrea que es una consecuencia tan general de las úlceras intestinales existía en sólo cinco de los casos observados. En trece, al contrario se comprobó una obstrucción a veces difícil de vencer".

Continúa el autor: "si los síntomas del sistema nervioso a veces no parecían tan intensos como lo son generalmente en el iliotifus, no faltaron en la mayoría de los casos. Eran frecuentes los desvarios o alucinaciones insignificantes, especialmente en las primeras noches. En casi todos los casos habia al principio agitación nerviosa con insomnio y después mucha apatía y somnolencia; había también casos en los cuales los enfermos durante casi toda la segunda y tercera semana permanecieron mudos y en que era difícil sacarlos de su apatía letárgica".

La enfermedad duró en los "casos felices" término medio 15 días y 11 en los casos fatales. La muerte más repentina ocurrió a los 5 días, la más tardía a los 31 , exceptuando siempre "los casos que parecen no haber sido de tifus".

En los 31 enfermos seguidos en forma ordenada por el doctor Martin, la edad osciló entre los 4 y 63 años, de los cuales fallecieron 2 , sin contar los cinco lactantes ya mencionados. Pero, como también se señaló más arriba, hubo un total de 28 casos fatales.

¿Cuáles fueron las medidas terapéuticas en estos enfermos? Escribió el doctor Martin lo siguiente: "para combatir la epidemia mi primer cuidado era mejorar los alimentos y el abrigo de los enfermos y convalecientes.
Frazadas de lana, harina, cervez a y vino, carne de ovejas $y$ de gallinas fueron distribuidas a los enfermos. Además segui una terapia sintomática dando purgantes a los que no obraban, calmando a veces la agitación nerviosa por narcóticos, haciendo un uso franco de la quina como antifebril'.

Concluyo -dice el doctor Martin- "para no ocupar demasiado el tiempo de la ilustre Sociedad y pido que juzgue con benevolencia estas observaciones hechas en una isla apartada de los centros cientificos y privada de todo aparato de medicina, de toda comodidad y hasta de muebles, que en otros lugares se consideran como esenciales para la vida humana"1,3,4.

\section{Comentario sobre las posibilidades diagnósticas}

La petición que hace el doctor Martin a la Sociedad Médica para "que los juzgue con benevolencia", habla muy bien de su honestidad y entereza moral y seguramente debió reconocerse su esfuerzo titánico con gran preocupación por la actividad médica que le tocó realizar en la isla Huar, no contando con la ayuda de otros médicos, siendo necesario señalar, que cuando ocurrió este gran problema de salud, el doctor Martin aún no cumplía un año de actividad en Chile.

Da la impresión que el propio doctor Martin dudó en algún momento que pudiera tratarse exclusivamente de fiebre tifoidea, con excepción de aquellos enfermos en los que encontró la presencia de roseolas tíficas y en los que junto con el síndrome febril hubo predominio de compromiso del aparato digestivo y manifestaciones septicémicas, con participación del sistema nervioso.

Si bien no se conoció el total de casos habidos, podría concluirse que un número indeterminado de pacientes sufrieron infecciones respiratorias agudas de diferentes tipos, incluso neumonía con gran pleurodinia, que indujo a los isleños a denominar la epidemia con el nombre de "puntada".

El comienzo brusco, con gran cefalea y alza febril importante y cierto grado de excitación psicomotora, aunque no existió constancia de exantema petequial o máculopapuloso generalizado, podría plantear la posibilidad diagnóstica de tifus exantemático epidémico o murino. Vale la pena señalar que el exantema puede estar ausente en $10 \%$ de los enfermos de tifus exantemático epidémico $y$ en $25 \%$ de los con tifus murino ${ }^{6}$.

En realidad todas son suposiciones. La inclemencia del otoño o del invierno de 1872 en la isla, avalaría la posibilidad de infecciones respiratorias agudas y las descripciones clínicas de otros enfermos llevarían a pensar en fiebre tifoidea.

En todo caso nunca podrá dilucidarse este misterio. 


\section{Resumen}

Se recuerda y comenta la publicación en el primer número de Revista Médica de Chile (1872-73) del doctor Carlos Martin, de una epidemia ocurrida en la isla de Huar en el seno de Reloncaví ( $X^{a}$ Región de Los Lagos, Chile), descrita como "epidemia tifoidea" y se expone la fisonomía clínica deduciendo la posibilidad de dos tipos de patologías: infecciones respiratorias agudas y fiebre tifoidea.

\section{Referencias}

1.- Martin, C. La epidemia tifoidea de la isla de Huar, provincia de Llanquihue. Rev. Méd. Chile. 1872-73, 1: 264-70.

2.- Laval M E. Biografías de médicos en Chile. Siglo XIX. (Inédito).

3.- Asta-Buruaga, C.F. Diccionario geográfico de la República de Chile. $2^{\text {a }}$ edición. Santiago de Chile. 1899.

4.- Riso-Patrón, L. Diccionario geográfico de Chile. Impta. Universitaria. Santiago de Chile. 1924.

5.- Diario El Día. La Serena, Chile. Domingo 27 de enero de 2008.

6.- Kraljevic O R. El tifus murino y otras rickettsiasis. Ed. Stanley. Santiago de Chile. 1957. 